\title{
ANTECEDENTES Y FORMACIÓN PROFESIONAL DEL OPTÓMETRA EN COLOMBIA
}

${ }^{1}$ Margarita María Ayala Cárdenas, ${ }^{2}$ Claudia Ximena Martínez Estupiñán,

'Optómetra ULS, Especialista Dacencia Universitaria UIS, docente U. Santo Tomás

${ }^{2}$ Optómetra ULS, Docente U. Santo Tomás

Autor responsable de correspondencia: Margarita Maria Ayala Cárdenas

e-mail:mayalaca@ustabuca.educo

\section{RESUMEN}

La llegada de los primeros optómetras al país, provenientes de escuelas Norteamericanas y Europeas, con formación en óptica y refracción, data de 1914. En 1933, se expiden en Colombia los primeros decretos (449 y 1291), que reglamentan la profesión en el territorio nacional.

En 1952 se crea la Federación Colombiana de Optómetras Graduados (Fedopto), agremiación que reúne a los profesionales con formación universitaria, para promover las actividades académicas y la defensa de la profesión y posteriormente en 1954, el Decreto Ley 0825 del 23 de Marzo, modifica su predecesor 1291, que define el campo de acción profesional.

Con este ajuste, el optómetra se constituye como un profesional independiente con proyección social definida como "...profesión dedicada a la determinación, medida y tratamiento de las alteraciones de la refracción, acomodación y movilidad del ojo humano. El ejercicio incluye la adaptación de lentes de contacto, prótesis oculares y la práctica de ejercicios ortópticos sin el uso de medicamentos o intervención quirúrgica». [Ayala MM, Martínez CX. Antecedentes y formación profesional del optómetra en Colombia. Ustasalud 2002;1:57-61]

\section{Background and Professional Training of Optometrist in Colombia}

\begin{abstract}
The arrival of the first optomertist to the country from American European Schools with optical and refraction studies, dates from 1914. In 1933, the first decrees (449 and 1291), that regulate the profession in Colombia, are issued for the whole National territory.

In 1952, is created the Colombian Graduated Optometrists Federation (Fedopto), wich reunited the professionals with graduated studies in order to promote the academic activities and the defense of the profession. After that, in 1954, the decreelaw 0825 from March 23th, modifies the other one, 1921, defending the profession's field of action.

With this one, the Optometry became an autonomous and independent profession with social projection, defined as "....profession devoted to determination and measurement of the refraction, accomodation and mobility defects of the human vision. The trial, prescription and adaptation of the lens that correct these defects. The conditioning of the contact lens, ocular protesics devices and the practice of orthoptic exercises, without using drugs, medicine or surgery».
\end{abstract}

En 1966 la Universidad de La Salle, por recomendación de un grupo de Optómetras federados funda la Facultad de Optometría, contando en sus inicios, con el respaldo de distinguidos profesores de las más importantes universidades de Bogotá.
A principios de los ochenta, la Facultad de Optometría hace su primer contacto con la comunidad, mediante la implementación de brigadas de salud visual, que posteriormente se convierten en planes y programas, como los 
desarrollados en diferentes Departamentos y Municipios del territorio nacional en asocio con autoridades regionales o instituciones como Plan Nacional de Rehabilitación, Fundación Restrepo Barco, Banco Social e INCI, que aunando esfuerzos han generado programas de alto impacto social con cobertura de importancia como Metrosalud en el área Metropolitana de Medellín, que decretó hace seis años, la cobertura universal para la población de 5 a 14 años.

Con el desarrollo de dichos programas, se genera información epidemiológica sustentada, originando interés por la problemática de salud visual como tema de Salud Pública; de esta forma se recopilan algunos de los estudios pertinentes que nos dan una dimensión del problema visual de la población en aras de la implementación de futuros trabajos en el área.

El Estudio Nacional de Salud, E.N.S. (1985) en su publicación "La Agudeza Visual en Colombia", basado en la investigación Nacional de Morbilidad (1965-1966), expuso el primer estudio específico sobre agudeza visual, mostrando que la disminución de agudeza visual es la tercera causa de morbilidad en la población en Colombia.

En 1990 el Estudio Sectorial de Salud, reconoce que: "Las enfermedades de los ojos ofrecen un enorme potencial para la prevención, pues los problemas de agudeza visual pueden ser detectados y corregidos inclusive en los preescolares con acciones de tamizaje en guarderías y escuelas". El Sistema Nacional de Información sobre Discapacidad, 1995, llevado a cabo en diez ciudades, muestra que el $23.6 \%$ de la población presenta alguna discapacidad, encontrando que las visuales son las de mayor prevalencia, y que representan el $16.31 \%$ de la población.

Si comparamos los datos del Estudio Sectorial de Salud que hacen referencia al uso de corrección de afecciones visuales en un $20.1 \%$ de la población afectada y el estudio de Discapacidad visual presente en un $64.2 \%$ de la población, entendemos la importancia de la Optometría en el cuidado de la salud visual comunitaria.

La Ley 100, de 1993, que basa su proyecto de atención en la promoción de la salud y la prevención de la enfermedad como sus elementos constitutivos fundamentales, define los niveles de complejidad en atención de enfermedades y buscan la mejor relación costo- efectividad en prestación de servicios de salud. Para esto requiere profesionales capacitados para el cubrimiento de poblaciones remotas y áreas marginales de las grandes ciudades y el país, garantiza la cobertura total en el tiempo definido por la ley. La reglamentación legal y planes de atención, contemplan actividades relacionadas con la salud visual general y particularmente las relacionadas con la promoción de salud y presencia de la enfermedad, asistencia y suministro de insumos ópticos para todos los sectores de la población; puede decirse, sin temor a equivocación, que actualmente todos los estratos sociales tienen acceso a atención preventiva visual por mandato legal.

Los programas de las Facultades de Optometría en Colombia, se han adaptado a las necesidades expuestas, enfatizan la formación del talento humano y científico, y ajustan los currículos académicos a los lineamientos legales de Salud en Colombia.

Conciente de los cambios en el sistema de salud generados por la Constitución Política de 1991, que posteriormente originaron la Ley 100, y basándose en los principios de universalidad, cobertura e integralidad del servicio de salud, la Optometría se ofrece al país como profesión integral enfocada a la resolución de problemas de Salud Visual, basada en el esquema universal del Cuidado Primario de la Salud, el cual permite el uso racional y el aprovechamiento de los recursos profesionales y fisicos, entrenan y capacitan los profesionales en las áreas específicas que manejan los problemas visuales de mayor incidencia mediante acciones preventivas, promocionales, de valoración y rehabilitación en estados tempranos y con tratamientos extra hospitalarios y/0 ambulatorios de cubrimiento masivo, bajo costo, alto impacto y eficacia comprobada.

Frente a estos conceptos, se plantea la realidad de la Optometría rural, materializada en la práctica extramural de estudiantes de último semestre y de los Optómetras, que vinculados a hospitales regionales, e instituciones privadas, cubren las necesidades del servicio de salud visual y ocular. Por lo tanto, se analiza la necesidad de formar un profesional bajo este esquema de salud, que responda eficientemente en primer y segundo nivel por la atención de necesidades de la población en forma oportuna que diagnostique y trate en forma eficaz las alteraciones visuales y oculares cuando aparezcan, y remita en forma precisa y oportuna aquellas que requieren otro tipo de manejo especializado. 
Con estos principios, se crea una nueva reglamentación en el ejercicio profesional de la Optometría, que se materializa en la Ley 372 de 1997, sancionada el día 28 de mayo por el presidente Ernesto Samper Pizano, y que reglamenta la Optometría de la siguiente forma: "La Optometría es una profesión de la salud que requiere títuIo de idoneidad universitario, está basada en una formación científica, técnica y humanistica. Su actividad incluye acciones de prevención y corrección de las enfermedades del ojo y del sistema visual por medio del examen, diagnóstico, tratamiento y manejo que conduzcan a lograr la eficiencia visual y la salud ocular, así como el reconocimiento y diagnóstico de las manifestaciones sistémicas que tienen relación con el ojo y que permiten preservar y mejorar la calidad de vida del individuo y de la comunidad".

Esta Ley se encuentra reglamentada por el Decreto Ley 1340 de 1998, que además de definir el perfil del Optómetra, consagra las actividades que debe realizar como profesional de la salud visual y estipula el grupo de medicamentos de uso tópico externo que puede manejar el Optómetra en el ejercicio de su profesión. Este decreto ley, liderado por los decanos de facultades de Optometría y asesores del gremio, reúne la experiencia de veinte años de la profesión en el territorio Colombiano.

De igual forma, la ley responde a las necesidades de la academia referidas a una formación dinámica, basada en la problemática de la salud, permite su actualización permanente e impulsa la creación de la Asociación de Facultades y Programas de Optometría (ASCOFAOP), ente que agrupa a Ia fecha cinco Facultades de Optometría aprobadas por el ICFES y cuyo objetivo es el desarrollo académico e intercambio entre las Facultades que lo componen.

\section{FORMACIÓN DEL OPTÓMETRA}

Conscientes de la necesidad de dar repuesta a los problemas de salud visual que requieren tratamiento oportuno, de actualizar y nivelar los conocimientos de acuerdo con los conceptos actuales de la Optometría, que actualmente se considera como profesión del área de la salud que se desarrolla bajo el esquema del cuidado primario de la Salud Visual (Ley 372 de 1997), se permitió que esta tuviera un plan de estudios adecuado con la formación del profesional integral de la salud visual que requiere la sociedad.
El profesional debe prepararse para resolver los problemas de sus pacientes, diagnosticar y tratar los problemas de la salud visual, bajo un criterio de integralidad que permita ser parte del bienestar bio-psico-social.

Dentro del currículum de toda Facultad de Optometría se generan CUATRO áreas básicas:

\section{ÁREA OPTOMÉTRICA}

Conocimientos básicos y especializados relacionados con la profesión. (Óptica de los lentes, refracción, Óptica Fisiológica, Lentes de Contacto, Ortóptica, Baja Visión, etc.).

\section{ÁREA DE CIENCIAS básicas}

El pilar fundamental en el desarrollo de la Optometría como una profesión INTEGRAL en el área de la salud, otorgando un soporte teórico que responda a las necesidades del Optómetra en su papel como el PROFESIONAL DEL CUIDADO PRIMARIO DE LA SALUD VISUAL.

Las ciencias básicas ofrecen los conceptos científicos referentes a la función óptica, visual y biológica, su organización sistémica biológica y la interrelación entre la función ocular y el organismo, la estructura ocular, sus funciones y patología, explorados en detalle con el fin de crear la base para entender las condiciones anómalas.

\section{ÁREA DE HUMANIDADES}

La Optometría, como ciencia del área de la salud, está dirigida al manejo de situaciones del ser humano o una comunidad específica, buscando mejorar su calidad de vida, con un soporte humanista que lo integre con la realidad socio-cultural del país y el mundo y fortalezca el objeto social de la profesión.

Se desarrollan conceptos básicos de la comunidad, formando al estudiante como administrador de programas de salud visual dirigidos hacia el área de la salud pública u ocupacional, con fundamentos gerenciales para su práctica privada 0 institucional.

\section{ÁREA DE CIENCIAS CLÍNICAS}

Desarrollo y conocimiento de la historia clínica integral, que afianza el desarrollo de destrezas clínicas y la correlación de datos. El manejo responsable de una historia clínica completa e integral agregado al análisis clínico formado en el estudiante, suministra las bases fundamentales en el manejo del paciente, permite solucionar su problema, y tiene en cuenta todo el manejo de la ética profesional que lleva consigo. 
El alumno debe desarrollar su práctica clínica, evaluando tres aspectos fundamentales:

a. Desempeño clínico.

b. Análisis clínico.

c. Manejo integral del paciente.

En el décimo semestre, se afianza el manejo integral mediante la práctica en diferentes campos de acción de la profesión. En el último semestre del ciclo de formación, el estudiante pone en práctica sus conocimientos, habilidades y destrezas en su trabajo EXTRAMURAL.

Desde su creación, toda facultad debe formar profesionales de salud cuyo ejercicio se ajuste a la reglamentación vigente en el sector y en su profesión; teniendo en cuenta las necesidades sociales, la cobertura con calidad y oportunidad en los servicios de salud, se forman profesionales en consonancia con las necesidades sociales, económicas, epidemiológicas y gremiales del país.

\section{LA FORMACIÓN DEL OPTÓMETRA}

El Optómetra debe ser un ser humano integral, con disposición de líder, objetivos dirigidos al desarrollo social, responsable del cuidado primario de la salud visual y capacitado en diseño y ejecución de actividades que le permitan mejorar y mantener las condiciones visuales y de salud ocular de su comunidad, con el apoyo de los niveles de atención e intervención.

El Optómetra Colombiano es educado para buscar respuesta a las necesidades individuales y colectivas de la comunidad basado en su dimensión investigativa, expresada a través de la capacidad de cuestionamiento y reflexión de su entorno. El Optómetra tiene un complejo desempeño profesional que reúne las siguientes actividades:

\section{ÁREA CLÍNICA}

Centrada en el cuidado de la salud visual y ocular del individuo. En esta área el Optómetra se prepara clínicamente para desempeñarse en el ejercicio de su profesión frente a dos aspectos fundamentales:

\section{a. Perfil Clínico Integral}

Se refiere a actividades como evaluación Optométrica Integral, tratamiento y control de alteraciones de agudeza visual y visión binocular; diseño, adaptación y control de lentes oftálmicos correctivos, terapéuticos o cosméti- cos; la aplicación de técnicas necesarias para el diagnóstico, pronóstico, tratamiento y rehabilitación de las anomalías de alteraciones visuales. También incluye remisiones y manejo interdisciplinario de alteraciones visuales con relación sistémica.

\section{b. Perfil Clínico Especializado}

Son actividades focalizadas en especialidades como Optometría Pediátrica, Geriátríca, Ortóptica y Pleóptica, entrenamiento en el uso de ayudas especiales de baja visión, contactología, patología ocular y exámenes especializados de electrodiagnóstico, pruebas campimétricas, paquimetría y topografía corneal computarizada entre otras.

\section{ÁREA DE SALUd PÚBLICA Y COMUNTTARIA}

Centrada en el cuidado de la salud visual y ocular aplicada a una población específica. En ésta área el optómetra se prepara clínicamente para desempeñarse en el ejercicio de su profesión en el campo comunitario, de la siguiente manera:

- Diseño, organización, ejecución y evaluación de políticas, planes, programas y proyectos, para la promoción, prevención, asistencia, rehabilitación y readaptación de problemas de la salud visual y ocular.

- Manejo de proyectos epidemiológicos, ocupacionales e investigativos de salud visual u ocular conducentes a la generación, adaptación o transferencia de tecnologías que permitan aumentar la cobertura, atención y suministro de soluciones para el control y rehabilitación de la función visual.

Dirección, administración y manejo de establecimientos de óptica para el suministro de insumos relacionados con la fase terapéutica visual.

La formación del profesional de optometría, a cargo de las instituciones de educación superior, debe procurar responder con calidad y eficiencia a las necesidades del contexto, brindando la mayor cobertura de servicios asistenciales, de prevención de la enfermedad y promoción de la salud visual, que contribuyan a mejorar la calidad de vida de la población.

Para tal fin, es necesario considerar no sólo el marco legal de seguridad social vigente, sino la dinámica de la salud en la población, que marcarán el derrotero en cuanto a la oferta de nuevos programas de posgrado que conduzcan a profundizar y avanzar en su campo de conocimiento. 


\section{BIBLIOGRAFÍA}

1. Decreto 0825 del 23 de Marzo de 1954

2. Decreto Ley 372 del 28 de Mayo de 1997.

3. Jaramillo J. Orlando: Por Qué y Para Qué Una Ley de Optometría. Revista Optometría, Bogotá, Septiembre de 1997.

4. FEDOPTO Seccional Antioquia. Memorias Congreso Nacional de Optometría. Agosto 1995.

5. Consejo Nacional de Acreditación (CNA). República de Colombia. Ministerio de Educación Nacional. Criterios y Procedimientos Para el Registro Calificado de Programas Académicos de Pregrado en Ciencias de la Salud. Bogotá, Julio de 2001 